\title{
Pädiatrische Fortbildung in Obergurgl 2022
}

Gespräch mit den beiden Tagungspräsidenten, Univ.-Prof. Dr. Wolfgang Sperl und Univ.-Prof. Dr. Daniel Weghuber.

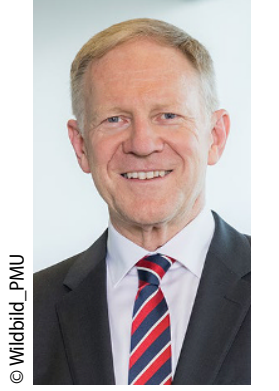

Univ.-Prof. Dr. Wolfgang Sperl

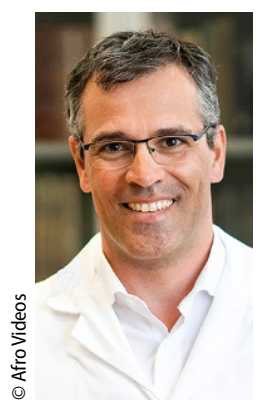

Univ.-Prof. Dr. Daniel Weghuber
Pädiatrie \& Pädologie: In Obergurgl treffen sich österreichische Pädiater traditionell im Jänner zur Fortbildung. Wodurch unterscheiden sich die beiden Veranstaltungen, das Internationale Pädiatrische Symposium Obergurgl und der Pädiatrische Fortbildungskurs Obergurgl?

Sperl: Der Fortbildungskurs im Anschluss an das Pädiatrische Symposium hat nun bereits eine 54jährige Tradition und verbindet länderübergreifend Deutschland, Schweiz, Österreich in einer breiten Fortbildung mit Up-To-Date Vorträgen in allen Subspezialitäten der Pädiatrie. Diese Fortbildung profitiert von der Schnittstelle mit dem Symposium. Zielgruppe vom Fortbildungskurs sind Kinderfachärztinnen und -ärzte sowohl aus dem niedergelassenen Bereich als auch Krankenhäusern und Universitätskliniken. Die Industrie ist auch immer wiederum interessiert, im Fortbildungskurs Neuerungen den Kinderfachärztinnen und -ärzten vorzustellen.

Weghuber: Seit 1974 treffen sich jedes Jahr Pädiaterinnen und Pädiater sowie Vertreterinnen und Vertreter assoziierter Disziplinen mit Entscheidungsträgern aus Politik, Industrie und NGOs beim Symposium in Obergurgl und diskutieren aktuelle Themen der pädiatrischen Ernährungs- und Präventionsmedizin. Das Symposium bietet den Rahmen zum Vorausdenken, wissenschaftliche Neuerungen und Trends, aber auch gesundheitspolitische "Hot Topics" werden aufgegriffen.

Beide Veranstaltungen haben letztes Jahr wegen der Pandemie mit SARS
CoV-2 pausiert. Haben Sie die Pause für die Veranstaltungen genutzt?

Sperl: Der Fortbildungskurs hat im Prinzip ein Jahr Pause gemacht. Heuer war dann der Entscheid für die Durchführung des Kurses. Die Anfrage ist ungebrochen, und der Kurs ist wiederum ausgebucht. In der Pause haben wir natürlich sehr viel über Digitalisierung und auch über die Möglichkeit von HybridVeranstaltungen gelernt.

Weghuber:Wir haben unseren Auftritt in den sozialen Medien aufgebaut und die Homepage verbessert (https://www.symposium-obergurgl.at). Auf unserer Facebook Seite werden regelmäßig aktuelle Beiträgen zu Präventions- und Ernährungsthemen in der Pädiatrie, sowie Auszügen aus dem Programm für die nächste Veranstaltung dargestellt (https://www. facebook.com/SymposiumObergurgl).

Sind Ihre beiden Tagungen reine Präsenztagungen, oder werden diese als Hybrid-Tagungen angeboten? Und warum?

Sperl \& Weghuber: Fortbildungskurs und Symposium sind als reine Präsenzveranstaltungen konzipiert, denn sie leben vom Diskurs. Eine Online-Veranstaltung würde jeweils dafür nicht den nötigen Rahmen bieten.

Sperl, ergänzend: ... und auch insgesamt den finanziellen Rahmen übersteigen.

Was ist neu, was ist 2022 anders als früher?

Sperl \& Weghuber: Der Fortbildungskurs bleibt bei seiner klassischen Tradition. Neu ist, dass das Symposium herangerückt ist und erstmalig auch im Veranstaltungszentrum "Gurgl Carat" stattfindet. Somit sind beide Veranstaltungen zentral am gleichen Ort, und etwaige Hygienekonzepte lassen sich besser umsetzen.

Wie lauten die Hauptthemen Ihrer Tagungen, wie ist Ihre Tagung gegliedert?

Sperl: Die Hauptthemen sind wie immer quer durch die Pädiatrie und auch benachbarte Fächer wie die Kinderchirurgie gestreut, am besten dem Programm zu entnehmen. Wie immer haben wir ein Kinder-Radiologie-Thema, auch das Thema, Klinik Hautnah" mit klinischen Fällen und ihren Lösungen. Besonders hervorheben möchte ich die personalisierte Präzisionsmedizin in der Pädiatrie, wo wir namhafte Referentinnen und Referenten gewinnen konnten. Unsere Tagung wird immer im Anschluss online gestellt und einzelne ausgewählte Vortragende werden u.a. auch eingeladen, ihre Beiträge zu publizieren.

Weghuber: Thematisch werden wir uns vier großen Themenblöcken widmen. Zum einen werden wir der Frage nachgehen, inwieweit die „personalisierte Ernährungsmedizin" in der Pädiatrie angekommen ist. Welche Möglichkeiten bietet eine individualisierte Frühgeborenen-Ernährung? Was bedeuten neueste Erkenntnisse der Muttermilchforschung für die Säuglingsernährung der Zukunft? Was versteckt sich hinter dem Begriff der Vesikel-Therapie, und kann diese in der Ernährungstherapie Anwendung finden?

Im zweiten Themenblock werden brandaktuelle Erkenntnisse zur Säuglings- und Kleinkinderernäh- 
rung sowie nationale Strategien zur Stillförderung und Chancen und Risiken der Beikost-Einführung besprochen.

In der dritten Sitzung erwartet uns ein spannendes Update zu ernährungsmedizinischen Aspekten der Allergieprävention.

Zu guter Letzt widmen wir uns dem brisanten Thema der Verhältnisprävention: Der Rolle von an Kinder gerichteter Werbung in unserer multimedialen Welt. Welche wissenschaftliche Evidenz gibt es, welche politischen Konsequenzen müssen gezogen werden, bzw. welche Maßnahmen zum Schutz unserer Kinder sind in konkreter Umsetzung?
Gibt es Ihren Kongress "zum Nachlesen" oder "Nachschauen"?

Weghuber: Wir konzentrieren uns immer auf ein Thema: Aus dem Jahr 2020 ist eine Publikation zum Thema Lebensmittel-Kennzeichnung (front-of-pack labelling) in Vorbereitung.

Was wünschen Sie sich für Ihre Tagung?

Sperl \& Weghuber: Wir wünschen uns eine Covid-freie geordnete Tagung, und dass wir uns in Präsenz wiedersehen können. Wir freuen uns auf einen Austausch, eine lebhafte Diskussion in angenehmer Atmosphäre mit hoher wissenschaftlicher Qualität.
Vielen Dank für das interessante Gespräch!

Das Interview führte Dr. Renate Lessky-Höhl, 17.11.2021

Hinweis des Verlags. Der Verlag bleibt in Hinblick auf geografische Zuordnungen und Gebietsbezeichnungen in veröffentlichten Karten und Institutsadressen neutral.

Paediatr. Paedolog. 2021 · 56:262-263 https://doi.org/10.1007/s00608-02100953-y

(c) Springer-Verlag GmbH Austria, ein Teil von Springer Nature 2021

Hier steht eine Anzeige. 